CERN-TH.7492/94

TIFR/TH/94-54

\title{
NON-EXISTENCE OF POSITIVE PHASE-SPACE DISTRIBUTION FUNCTIONS IN QUANTUM MECHANICS
}

\author{
A. Martin and S.M. Roy*) \\ Theoretical Physics Division, CERN \\ CH-1211 Geneva 23
}

\begin{abstract}
We show that in space dimension $\geq 2$, there cannot exist a positive definite phase-space distribution function whose marginals coincide with the quantum position probability density, momentum probability density and joint probability densities of commuting components of position and momentum.
\end{abstract}

*) On leave from (and address after 4 November 1994) Tata Institute of Fundamental Research, Homi Bhabha Road, Bombay 400 005, India.

CERN-TH.7492/94

TIFR/TH/94-54

October 1994 



\section{$1 \quad$ Introduction}

A phase-space distribution function was first introduced in quantum mechanics by E. Wigner [1] to calculate the quantum correction for thermodynamic equilibrium. Since then, it has found wide applications in many branches of physics [2]. The Wigner distribution $W(\vec{x}, \vec{p}, t)$ for a quantum state with wave function $\psi(\vec{x}, t)$ is defined as

$$
W(\vec{x}, \vec{p}, t)=\int \frac{d \vec{y}}{(2 \pi \hbar)^{n}} \psi^{*}\left(\vec{x}+\frac{\vec{y}}{2}, t\right) \psi\left(\vec{x}-\frac{\vec{y}}{2}, t\right) \exp \left(i \vec{p} \cdot \frac{\vec{y}}{\hbar}\right)
$$

where $\vec{x}$ is an $n$-dimensional position coordinate, and $\vec{p}$ the corresponding momentum. The property most crucial for applications is that the marginals of the Wigner distribution coincide exactly with the quantum position and momentum probability densities:

$$
\begin{aligned}
& \int W(\vec{x}, \vec{p}, t) d \vec{p}=|\psi(\vec{x}, t)|^{2}, \\
& \int W(\vec{x}, \vec{p}, t) d \vec{x}=|\tilde{\psi}(\vec{p}, t)|^{2},
\end{aligned}
$$

where we choose units $\hbar=1$, and denote by $\tilde{\psi}$ the Fourier transform of the wave function $\psi$. This property implies that the normal probability calculation using $W$ reproduces the correct quantum expectation values of a sum of a function of $\vec{x}$ and a function of $\vec{p}$ :

$$
\int d \vec{x} d \vec{p}[f(\vec{p})+g(\vec{x})] W(\vec{x}, \vec{p}, t)=\int \psi^{*}(\vec{x}, t)[f(-i \hbar \vec{\nabla})+g(\vec{x})] \psi(\vec{x}, t) d \vec{x} .
$$

However, $W$ cannot be interpreted as a joint probability density for $\vec{x}$ and $\vec{p}$ because it is not positive definite; e.g. the integral

$$
\int d \vec{x} d \vec{p} W_{\psi}(\vec{x}, \vec{p}, t) W_{\phi}(\vec{x}, \vec{p}, t)=\frac{|(\psi, \phi)|^{2}}{(2 \pi)^{n}}
$$

vanishes for two orthogonal states $\psi, \phi$. Moreover, Wigner showed that there does not exist a distribution, bilinear in $\psi$, which satisfies Eq. (4) and is everywhere (i.e. for all $\vec{x}, \vec{p}$ ) positive. There has been extensive work on the construction of smeared Wigner functions to obtain positivity [3], [2]; however, one then loses the exact validity of Eq. (4).

It has been noted [4] that the uncertainty principle does not preclude the existence of a positive definite phase-space density $\rho(\vec{x}, \vec{p}, t)$ obeying

$$
\rho(\vec{x}, \vec{p}, t) \geq 0, \quad \int \rho d \vec{p}=|\psi(\vec{x}, t)|^{2}, \quad \int \rho d \vec{x}=|\tilde{\psi}(\vec{p}, t)|^{2}
$$

Once the condition of bilinearity in $\psi$ is dropped, the solution $\rho=|\psi(\vec{x}, t)|^{2} \cdot|\tilde{\psi}(\vec{p}, t)|^{2}$, and an infinite number of other solutions were obtained by Cohen and Zaparovanny [4]. Recently, Roy and Singh [5] have constructed a deterministic quantum mechanics for one particle in one space dimension by imposing (6) and the additional condition of Hamiltonian evolution of the 
phase-space points (which leads to the Liouville condition $d \rho / d t=0$ ). The physical interpretation of $\rho$ thus achieved in one-dimensional configuration space encourages us to ask whether further physical conditions in addition to (6) can be imposed on $\rho$ in higher dimensions. In higher-dimensional configuration space (dimension $=$ space dimension $\times$ number of particles) quantum mechanics predicts not only the position and momentum probability densities occurring in (6) but also the joint probability densities for commuting components of position and momentum. For instance, $\left|\psi_{2}\left(x_{1} p_{2}\right)\right|^{2},\left|\psi_{1}\left(p_{1} x_{2}\right)\right|^{2}$, where the subscripts in $\psi_{1}, \psi_{2}$ denote partial Fourier transforms with respect to $x_{1}$ and $x_{2}$, respectively, are such quantum predictions in two dimensions, with

$$
\psi_{1}\left(p_{1} x_{2}\right)=\frac{1}{\sqrt{2 \pi}} \int_{-\infty}^{\infty} d x_{1} e^{-i p_{1} x_{1}} \psi\left(x_{1} x_{2}\right)
$$

and

$$
\psi_{2}\left(x_{1} p_{2}\right)=\frac{1}{\sqrt{2 \pi}} \int_{-\infty}^{\infty} d x_{2} e^{-i p_{2} x_{2}} \psi\left(x_{1} x_{2}\right) .
$$

(The dependence of $\psi$ and $\rho$ on time will not be explicitly indicated henceforth, since we are considering a fixed time.) Should we not require that, in addition to (6), $\rho$ satisfies also

$$
\int \rho d x_{1} d p_{2}=\left|\psi_{1}\left(p_{1} x_{2}\right)\right|^{2}, \quad \int \rho d p_{1} d x_{2}=\left|\psi_{2}\left(x_{1} p_{2}\right)\right|^{2} ?
$$

Further, should we not require that analogous conditions hold for arbitrary choice of coordinates $x_{1}, x_{2}$ ? If we define coordinates $x_{1 \alpha}, x_{2 \alpha}$ obtained from $x_{1}, x_{2}$ by an arbitrary rotation of coordinate axes by angle $\alpha$, and momenta $p_{1 \alpha}, p_{2 \alpha}$ related similarly to $p_{1}, p_{2}$

$$
\left(\begin{array}{c}
x_{1 \alpha} \\
x_{2 \alpha}
\end{array}\right)=R\left(\begin{array}{c}
x_{1} \\
x_{2}
\end{array}\right),\left(\begin{array}{c}
p_{1 \alpha} \\
p_{2 \alpha}
\end{array}\right)=R\left(\begin{array}{c}
p_{1} \\
p_{2}
\end{array}\right)
$$

where

$$
R=\left(\begin{array}{cc}
\cos \alpha & \sin \alpha \\
-\sin \alpha & \cos \alpha
\end{array}\right)
$$

then $\psi$ and $\rho$ can be re-expressed in these new coordinates

$$
\begin{gathered}
\psi\left(x_{1}, x_{2}\right)=\psi_{\alpha}\left(x_{1 \alpha}, x_{2 \alpha}\right) \\
\rho\left(x_{1}, x_{2}, p_{1}, p_{2}\right)=\rho_{\alpha}\left(x_{1 \alpha}, x_{2 \alpha}, p_{1 \alpha}, p_{2 \alpha}\right) .
\end{gathered}
$$

In addition to conditions (6), which are invariant under coordinate rotations, should we not require that for arbitrary angle $\alpha$,

$$
\int \rho_{\alpha} d x_{1 \alpha} d p_{2 \alpha}=\left|\psi_{1 \alpha}\left(p_{1 \alpha}, x_{2 \alpha}\right)\right|^{2}, \quad \int \rho_{\alpha} d p_{1 \alpha} d x_{2 \alpha}=\left|\psi_{2 \alpha}\left(x_{1 \alpha}, p_{2 \alpha}\right)\right|^{2} .
$$

Here $\psi_{1 \alpha}, \psi_{2 \alpha}$ denote partial Fourier transforms of $\psi_{\alpha}\left(x_{1 \alpha}, x_{2 \alpha}\right)$ with respect to $x_{1 \alpha}$ and $x_{2 \alpha}$ respectively and are defined similarly to $(7),(8)$.

It is an easy exercise to check that conditions (6) except positivity of $\rho$, and conditions (13) are compatible. The Wigner distribution is an example of such a $\rho$. We now prove that with positivity of $\rho$ added, there is an insurmountable obstruction. 


\section{A Theorem}

There exist wave functions in a configuration space of dimension $\geq 2$ for which it is impossible to find a non-negative phase-space distribution whose marginals coincide exactly with all quantum joint probability densities for component of position and a commuting component of momentum.

To prove this for a configuration space of dimension 2 , it is sufficient to find a $\psi$ for which Eqs. (13) cannot hold for all $\alpha$ with a non-negative phase-space density $\rho$. Consider the normalized wave function

$$
\psi\left(x_{1}, x_{2}\right)=\mu^{3} N\left(x_{1}^{2}+x_{2}^{2}-\frac{c}{\mu^{2}}\right) \exp \left[-\mu^{2}\left(x_{1}^{2}+x_{2}^{2}\right)\right],
$$

with

$$
N=\sqrt{\frac{2}{\pi}}\left[\left(c-\frac{1}{2}\right)^{2}+\frac{1}{4}\right]^{-1 / 2} .
$$

This implies that

$$
\begin{aligned}
\psi_{\alpha}\left(x_{1 \alpha}, x_{2 \alpha}\right) & =\mu^{3} N\left(x_{1 \alpha}^{2}+x_{2 \alpha}^{2}-\frac{c}{\mu^{2}}\right) \exp \left[-\mu^{2}\left(x_{1 \alpha}^{2}+x_{2 \alpha}^{2}\right)\right], \\
\psi_{1 \alpha}\left(p_{1 \alpha}, x_{2 \alpha}\right) & =\frac{N}{\sqrt{2}}\left(\mu^{2} x_{2 \alpha}^{2}-\frac{p_{1 \alpha}^{2}}{4 \mu^{2}}+\frac{1}{2}-c\right) \exp \left[-\left(\mu^{2} x_{2 \alpha}^{2}+\frac{p_{1 \alpha}^{2}}{4 \mu^{2}}\right)\right], \\
\psi_{2 \alpha}\left(x_{1 \alpha}, p_{2 \alpha}\right) & =\frac{N}{\sqrt{2}}\left(\mu^{2} x_{1 \alpha}^{2}-\frac{p_{2 \alpha}^{2}}{4 \mu^{2}}+\frac{1}{2}-c\right) \exp \left[-\left(\mu^{2} x_{1 \alpha}^{2}+\frac{p_{2 \alpha}^{2}}{4 \mu^{2}}\right)\right], \\
\tilde{\psi}\left(p_{1}, p_{2}\right) & =\frac{N}{2 \mu}\left(1-c-\frac{p_{1}^{2}+p_{2}^{2}}{4 \mu^{2}}\right) \exp \left[-\left(\frac{p_{1}^{2}+p_{2}^{2}}{4 \mu^{2}}\right)\right] .
\end{aligned}
$$

Choosing $c=1 / 2$, we see that $\psi_{2 \alpha}\left(x_{1 \alpha}, p_{2 \alpha}\right)$ vanishes on the surfaces $4 \mu^{4} x_{1 \alpha}^{2}-p_{2 \alpha}^{2}=0$, i.e., on the surfaces

$$
x_{1} \cos \alpha+x_{2} \sin \alpha= \pm \frac{1}{2 \mu^{2}}\left(p_{2} \cos \alpha-p_{1} \sin \alpha\right) .
$$

From the second of Eqs. (13), if $\rho_{\alpha}$ is non-negative everywhere, there is no possibility of cancellations between different parts of the phase space, hence

$$
\rho\left(x_{1}, x_{2}, p_{1}, p_{2}\right)=0
$$

whenever Eq. (16) is satisfied for any value of $\alpha$. For any value of $\left(x_{1}, x_{2}, p_{1}, p_{2}\right)$ and any sign + or - on the right-hand side of Eq. (16), this gives a real value for $\tan \alpha$; e.g. for + sign,

$$
\tan \alpha=\frac{p_{2}-2 \mu^{2} x_{1}}{p_{1}+2 \mu^{2} x_{2}} .
$$


Thus, for any $\left(x_{1}, x_{2}, p_{1}, p_{2}\right)$ we can find $\alpha \in[0, \pi]$ such that (16) is satisfied, and conclude that $\rho$ must vanish identically all over phase space. This is of course contradictory to the assumed validity of Eqs. (13), and thus the theorem is proved for two-dimensional configuration spaces.

It is trivial to extend it to higher dimensions. For example in three dimensions, start from

$$
\psi\left(x_{1}, x_{2}, x_{3}\right)=\psi\left(x_{1}, x_{2}\right) \phi\left(x_{3}\right),
$$

where $\psi\left(x_{1}, x_{2}\right)$ is given by (14) and $\phi$ is an arbitrary normalized function. The equation

$$
\int \rho_{\alpha} d p_{1 \alpha} d x_{2 \alpha} d p_{3 \alpha}=\left|\psi_{2 \alpha}\left(x_{1 \alpha}, p_{2 \alpha}\right)\right|^{2}\left|\phi\left(x_{3}\right)\right|^{2}
$$

and the previous two-dimensional argument then requires $\rho$ to vanish identically in the sixdimensional phase space, thus proving the theorem.

In the proof of the theorem the crucial fact was the vanishing of the partial Fourier transforms $\psi_{2 \alpha}\left(x_{1 \alpha}, p_{2 \alpha}\right)$ on the hyperplanes (16), leading to the absence of a classical phase-space density.

\section{Physical Meaning of the Theorem}

(i) The present procedure of using the rotationally symmetric wave function (14) and imposing conditions (13) for a continuum of values of $\alpha$ is somewhat reminiscent of the proof of Bell's theorem [6] on the impossibility of local hidden variable theories; there one uses a singlet state of two spin-half particles and spin correlations in four different experimental configurations. Nevertheless, the present theorem must in general have a different interpretation from Bell's theorem, because the phase-space density $\rho(\vec{x}, \vec{p})$ for one $\vec{x}$ has been allowed to have arbitrary dependence on the wave function at all points of space. We do not impose locality.

(ii) The correlated normalizable wave function (14) might seem analogous to the correlated unnormalizable wave function used by Einstein-Podolsky-Rosen [7] in arguing incompleteness of quantum mechanics. However, the essence of the present theorem is different from the EPR argument. For example, it is known [8] that the EPR wave function leads to a positive definite Wigner function, which obeys all the conditions we imposed on $\rho(\vec{x}, \vec{p})$.

(iii) Does the theorem mean that the real existence of a phase-space probability density in $a$ given experimental situation is contradictory to quantum mechanics? It seems not. Quantum mechanics requires that in an experiment measuring $x_{1 \alpha}, p_{2 \alpha}$ the observed probability density be $\left|\psi_{2 \alpha}\left(x_{1 \alpha}, p_{2 \alpha}\right)\right|^{2}$; but in this experiment quantum mechanics does not ascribe any real existence to $x_{1 \beta}, p_{2 \beta}$ for $\beta \neq \alpha$, since that would in general require a different experiment due to non-commutation of $x_{1 \alpha}$ and $p_{2 \beta}$. Thus, without violating quantum mechanics, the theorem allows realistic but context-dependent (i.e. experiment-dependent) phase-space densities. The theorem shows that a context-independent phase-space density will conflict with quantum mechanics. 
(iv) The present theorem can be compared with the well-known one of Gleason, of Kochen and of Specker [9] (GKS). The GKS theorem asserts the impossibility of non-contextual deterministic hidden variable theories for a quantum system whose state space has dimensionality greater than two. The GKS non-contextuality assumption responsible for the conflict with quantum mechanics (as pointed out by Bell [9]) is the following: if a projection operator $P_{1}$ is part of two different complete commuting sets $\left(1=P_{1}+P_{2}+P_{3} \ldots, 1=\right.$ $\left.P_{1}+P_{2}^{\prime}+P_{3}^{\prime} \ldots\right)$ the result of measuring $P_{1}$ is independent of which complementary set, $P_{2} \ldots$ or $P_{2}^{\prime} \ldots$ is measured at the same time. In spite of the great mathematical difference between the present theorem and the GKS theorem, both seem to bring out the context dependence of reality in quantum mechanics.

(v) The theorem makes no use of $\psi$ being complex. Hence it proves the non-existence of positive definite joint intensity distribution in position and wave number for any wave phenomenon (sound waves, electromagnetic waves in signal processing, etc.).

It is possible to obtain a quantitative measure of quantum violation of non-contextuality of phase-space probability density by developing probability sum rules that follow from this non-contextuality. It is also possible to develop the work of Ref. [5] in higher dimensions by taking the contextuality of phase-space density into account. These results will be reported separately.

One of us (SMR) wishes to warmly thank Virendra Singh for an ongoing collaboration which originated the question answered here, John Ellis for the invitation to CERN, and the French Ministry of Foreign Affairs for travel support.

\section{References}

[1] E. Wigner, Phys. Rev. 40 (1932) 749.

[2] N.L. Balazs and B.K. Jennings, Phys. Rep. 104 (1984) 347;

M. Hillery, R.F. O’Connell, M.O. Scully and E.P. Wigner, Phys. Rep. 106 (1984) 121.

[3] K. Husimi, Proc. Phys. Math. Soc. Japan 22 (1940) 264;

N.D. Cartwright, Physica 83A (1976) 210;

R.F. O'Connell and E.P. Wigner, Phys. Lett. 86A (1981) 121;

J.J. Halliwell, Phys. Rev. D46 (1992) 1610.

[4] L. Cohen and Y.I. Zaparovanny, J. Math. Phys. 21 (1980) 794;

L. Cohen, J. Math. Phys. 25 (1984) 2402. 
[5] S.M. Roy and V. Singh, "Deterministic Quantum Mechanics in One Dimension", to appear in the Proceedings of the Conference "Bose and Twentieth Century Physics", Calcutta, January 1994 (Kluwer Academic, Dordrecht, Netherlands), and CERN preprint TH.7481/94, hep-ph/9410378.

[6] J.S. Bell, Physics 1 (1964) 195.

[7] A. Einstein, B. Podolsky and N. Rosen, Phys. Rev. 47 (1935) 777.

[8] J.S.Bell, "EPR correlations and EPW distributions", in "New Techniques and Ideas in Quantum Measurement Theory" (New York Academy of Sciences, 1986).

[9] A.M. Gleason, J. Math. Mech. 6 (1957) 885;

S. Kochen and E.P. Specker, J. Math. Mech. 17 (1967) 59;

J.S. Bell, Rev. Mod. Phys. 38 (1966) 447. 